\title{
Das Institut für Sächsische Geschichte und Volkskunde e. V. in Dresden Bericht für das Jahr 2016
}

von

ENNO BÜNZ

„Sachsen: weltoffen!“ - unter den zahlreichen Publikationen, die das Institut für Sächsische Geschichte und Volkskunde im Berichtsjahr herausgegeben hat, wird dieser Titel wohl als besonders einprägsam in Erinnerung bleiben. In dieser Veröffentlichung haben die Mitarbeiterinnen und Mitarbeiter des Instituts mit fundierten wissenschaftlichen Beiträgen aus der laufenden Arbeit nicht nur zu aktuellen Debatten beigetragen, sondern sie haben auch deutlich gemacht, dass unsere Einrichtung seit mittlerweile fast zwanzig Jahren einen erfolgreichen Weg zwischen wissenschaftlicher Grundlagenarbeit und Vermittlung landesgeschichtlichen und volkskundlichen Wissens über Sachsen und seine Nachbarregionen beschreitet.

Der vorliegende Tätigkeitsbericht legt Rechenschaft über drei gemeinsame Vorhaben des Instituts sowie jeweils zehn Vorhaben der Bereiche Geschichte und Volkskunde ab. Das Themenspektrum ist, wie mittlerweile schon selbstverständlich, breit angelegt und reicht in beiden Bereichen von Vorhaben der Grundlagenforschung und entsprechend langfristig angelegten Projekten über Promotionsvorhaben, die stets nur wenige Jahre laufen, bis hin zu aktuellen Themen, die im Bereich Geschichte etwa durch das Reformationsjubiläum 2017 bestimmt sind, im Bereich Volkskunde hingegen durch die nächste Sächsische Landesausstellung zur Industriekultur. Es spricht für die Themenbreite des Instituts, dass seine Mitarbeiterinnen und Mitarbeiter für beide Anlässe gerüstet sind: Im Bereich Geschichte wird an der Edition der Fürstinnenkorrespondenzen der Reformationszeit gearbeitet, und im Berichtsjahr ist der zweite Band dieses Vorhabens abgeschlossen worden. Im Bereich Volkskunde wurde in den letzten Jahren eine Monografie über die Industriestadt Plauen 1880 bis 1933 erarbeitet, die in diesem Jahr fertiggestellt werden konnte. Neue thematische Perspektiven eröffnet im Bereich Geschichte das auf die Geld- und Finanzpolitik des 18. Jahrhunderts ausgerichtete Projekt „Das Geld in Krisenzeiten“, das im Berichtsjahr mit einer Tagung begonnen wurde. Als drittmittelfinanziertes Vorhaben wurde im Bereich Volkskunde das Kontaktzonenprojekt erfolgreich fortgesetzt, das mit seiner Ausrichtung auf den deutsch-tschechisch-polnischen Grenzraum zugleich deutlich macht, dass das Institut auch international vernetzt arbeitet und neben dem sächsischen Arbeitsbereich auch die Nachbarregionen mit im Blick hat. Das Internet-Angebot des Instituts wird laufend ausgebaut. Exemplarisch sei hervorgehoben, dass im Berichtsjahr das Digitale Historische Ortsverzeichnis von Sachsen mit der Erschließung der sächsischen Gerichtsbücher im Umfang von 25925 Bänden verknüpft werden konnte.

Diese Vorhaben seien hier stellvertretend für viele andere erwähnt, die 2016 gut vorangekommen sind und deren Ergebnisse in künftigen Tätigkeitsberichten zu thematisieren sind. Darüber hinaus haben sich die Mitarbeiterinnen und Mitarbeiter des Instituts durch Publikationen und Vorträge, durch Mitwirkung in Gremien und Kommissionen, aber auch durch Lehre an Universitäten ausgewiesen. Wie immer ist viel Arbeitskraft in die laufende Betreuung unserer digitalen Publikationen im Internet 
geflossen, die weniger sichtbar ist als der Arbeitsertrag unserer gedruckten Veröffentlichungen, der sich 2016 in neun Bänden unserer Schriftenreihen sowie in den beiden Jahrgängen unserer Zeitschriften niedergeschlagen hat.

Die Erfolgsbilanz ist umso eindrucksvoller, weil sie in einem Jahr erzielt wurde, das durch zahlreiche Personalwechsel und damit einhergehende Stellenvakanzen geprägt war, aber auch durch Engpässe des Haushalts, die an die Grenze der Belastbarkeit gingen. Ungeachtet dieser Umstände waren im Berichtsjahr die Vorbereitung der Evaluation des Instituts 2017 und die Prüfung durch den Landesrechnungshof zu bewältigen. Im Ergebnis hat der Landesrechnungshof keine grundlegende Kritik an der Arbeit des Instituts geäußert. Vielmehr ist aus unserer Sicht vor allem die Forderung an den $\mathrm{Zu}$ wendungsgeber hervorzuheben, für eine angemessene Ausfinanzierung des Instituts zu sorgen. Dass dies mit dem Doppelhaushalt 2017/18 tatsächlich gegeben ist, betrachten wir als Anerkennung der Arbeit unserer Einrichtung, die seit 1997 im Dienste Sachsens steht.

\section{Forschungsprojekte 2016}

\section{Gemeinsame Projekte der Bereiche Geschichte und Volkskunde}

Schriften zur sächsischen Geschichte und Volkskunde; Quellen und Materialien zur sächsischen Geschichte und Volkskunde; Bausteine zur sächsischen Geschichte und Volkskunde; Spurensuche. Geschichte und Kultur Sachsens. Projektleitung: Direktorium/Bereichsleiterinnen, Projektbearbeitung: Direktorium/Bereichsleiterinnen/ wissenschaftliche Mitarbeiter.

Sachsen: weltoffen! Projektleitung: Direktorium/Bereichsleiterinnen, Projektbearbeitung: Ira Spieker, Kollegium des ISGV, URL: http://www.isgv.de/aktuelles/sachsenweltoffen

Erinnerungslandschaften: Transformationsprozesse im ländlichen Raum Ostdeutschlands - öffentliche Diskurse und individuelle Wahrnehmungen (von 1945 bis in die Gegenwart). Projektleitung: Martina Schattkowsky, Ira Spieker. Projektbearbeitung: Lutz Vogel (bis 6/2016), Uta Bretschneider (bis 11/2016), Henrik Schwanitz (ab 9/2016), N. N.

\section{Projekte des Bereichs Geschichte}

Sächsische Biografie. Projektleitung: Martina Schattkowsky, Projektbearbeitung: Martina Schattkowsky, Frank Metasch, Lutz Vogel (bis 6/2016), Henrik Schwanitz (ab 9/2016).

Fürstinnenkorrespondenzen der Reformationszeit. Briefedition der Herzogin Elisabeth von Sachsen, Band 2 (1533-1534). Projektleitung: Martina Schattkowsky, Projektbearbeitung: Jens Klingner.

Codex diplomaticus Saxoniae. Das Urkundenbuch der Stadt Dresden, Band 1. Projektleitung: Enno Bünz, Projektbearbeitung: Ulrike Siewert, Stefan Petersen (SAW).

Sächsisches Klosterbuch. Klöster, Stifte und Komtureien in Sachsen vor der Reformation. Projektleitung: Enno Bünz, Projektbearbeitung: Sabine Zinsmeyer (SAW). 
Zwischen Migration und Assimilation. Adel im sächsisch-böhmischen Grenzraum (16./17. Jahrhundert). Dissertationsprojekt. Projektleitung: Martina Schattkowsky, Projektbearbeitung: Martin Arnold.

Für Gott und Vaterland - Patriotismus und Militärdienst in Sachsen 1806 bis 1866/67. Dissertationsprojekt. Projektleitung: Winfried Müller, Projektbearbeitung: Torsten Schwenke.

Von der Natur gerahmt. Die Idee der „natürlichen Grenzen“ als Identitätsressource um 1800. Dissertationsprojekt. Projektleitung: Winfried Müller, Projektbearbeitung: Henrik Schwanitz.

Finanz- und Geldgeschichte Sachsens im 18. Jahrhundert. Projektleitung: Martina Schattkowsky, Projektbearbeitung: Frank Metasch.

Die Ratsherren gegen das Haus Habsburg. Die Sequestration der Klostergüter infolge der Reformation am Beispiel der Oberlausitz. Projektleitung: Winfried Müller, Enno Bünz, Projektbearbeitung: Petr Hrachovec (Tschechische Akademie der Wissenschaften, Prag).

Neues Archiv für sächsische Geschichte. Projektleitung: Karlheinz Blaschke, Enno Bünz, Winfried Müller, Martina Schattkowsky, Uwe Schirmer. Projektbearbeitung: Frank Metasch (Schriftleitung), Lutz Vogel (Rezensionen) (bis 6/2016), Jens Klingner (ab 9/2016).

\section{Projekte des Bereichs Volkskunde}

Lebensgeschichtliches Archiv für Sachsen. Projektleitung: Ira Spieker, Projektbearbeitung: Sönke Friedreich, Nadine Kulbe.

Visuelle Quellen zur Volkskultur in Sachsen. Das Bildarchiv des ISGV. Projektleitung: Andreas Martin, Ira Spieker, Projektbearbeitung: Andreas Martin, Hendrik Kellner.

Kontaktzonen. Kulturelle Praktiken im deutsch-tschechisch-polnischen Grenzraum. Projektleitung: Ira Spieker, Projektbearbeitung: Sarah Kleinmann, Uta Bretschneider (bis 11/2016), Arnika Peselmann (ab 11/2016), Ira Spieker.

An der Elbe. Das Leben mit dem Fluss. Projektleitung: Andreas Martin, Ira Spieker, Projektbearbeitung: Andreas Martin.

Erinnern an die Arbeit im Kollektiv. Brigadeleben in der DDR und seine postsozialistischen Tradierungen. Dissertationsprojekt. Projektleitung: Ira Spieker, Projektbearbeiterin: Merve Lühr.

Zwischen Aufstieg und Krise. Städtische Identität und Selbstwahrnehmung in Plauen, 1880-1933. Projektleitung: Ira Spieker, Projektbearbeitung: Sönke Friedreich.

Neue Sichtweisen. Zum Aufleben einer Aussichtsturm-Begeisterung. Projektleitung: Andreas Martin, Ira Spieker, Projektbearbeitung: Andreas Martin. 
Die deutsche Künstlersteinzeichnung ca. 1895-1918. Farblithographien als Bildwandschmuck für Schule und Haus. Projektleitung: Winfried Müller, Projektbearbeitung: Winfried Müller.

Erschließung und Digitalisierung des Nachlasses von Adolf Spamer. Projektleitung: Ira Spieker, Projektbearbeitung: Nadine Kulbe.

Volkskunde in Sachsen (Jahrbuch). Projektleitung: Ira Spieker, Sönke Friedreich, Projektmitarbeit: Mitarbeiterinnen und Mitarbeiter des Bereichs.

\section{Mitwirkung an Ausstellungen und Ausstellungskonzeptionen}

Zwischen Tradition und Modernität - Zeugnisse sächsischer Adelskultur (Arbeitstitel), Vorbereitung einer Dauerausstellung zur Geschichte des sächsischen Adels, Schloss Nossen. Konzeption und Organisation: Martina Schattkowsky.

\section{Tagungen und Workshops 2016}

Arbeiten im Kollektiv. Politische Praktiken der Normierung und Gestaltung von Gemeinschaft, Sächsisches Staatsarchiv - Staatsarchiv Chemnitz, 2. und 3. Juni 2016. Konzeption und Organisation: Merve Lühr.

Normalfall Migration? Kulturelle, historische und aktuelle Dimensionen, Deutsches Hygiene-Museum Dresden, Kulturrathaus Dresden und Universität Wrocław, 7. bis 9. Juni 2016. Organisation: Ira Spieker.

Kontaktzonen. Alternative Wissensvermittlung, Europäisches Zentrum der Künste, Hellerau, 18. und 19. November 2016. Konzeption und Organisation: Ira Spieker, Sarah Kleinmann, Uta Bretschneider.

Das Geld in Krisenzeiten. Geld- und finanzpolitische Innovationen des Siebenjährigen Krieges, Residenzschloss Dresden, 18. und 19. November 2016. Konzeption und Organisation: Frank Metasch, Rainer Grund (SKD).

\section{Publikationen 2016}

Neues Archiv für sächsische Geschichte, hrsg. von Karlheinz Blaschke, Enno Bünz, Winfried Müller, Martina Schattkowsky, Uwe Schirmer. Redaktion: Frank Metasch (Schriftleitung), Lutz Vogel (Rezensionen), Band 87 (2016), Neustadt a. d. Aisch: Verlag Ph. C. W. Schmidt.

Volkskunde in Sachsen, hrsg. vom Institut für Sächsische Geschichte und Volkskunde e. V. Schriftleitung: Ira Spieker, Sönke Friedreich, unter Mitarbeit von Uta Bretschneider u. a., Band 28 (2016), Dresden: Thelem Universitätsverlag.

Schriften zur sächsischen Geschichte und Volkskunde, hrsg. von Enno Bünz, Winfried Müller, Martina Schattkowsky, Ira Spieker, Leipzig: Leipziger Universitätsverlag: Band 49: EnNo Bünz/Ulrike Höroldt/Christoph Volkmar, Adelslandschaft Mitteldeutschland. Die Rolle des landsässigen Adels in der mitteldeutschen 
Geschichte (15.-18. Jahrhundert), 2016; zugleich erschienen als: Veröffentlichungen der staatlichen Archivverwaltung des Landes Sachsen-Anhalt, Reihe A, Band 22.

Band 53: Uta Bretschneider, „Vom Ich zum Wir?“. Flüchtlinge und Vertriebene als Neubauern in der LPG, 2016.

Band 54: Dirk Martin Mütze, Das Augustiner-Chorherrenstift St. Afra in Meißen (1205-1539), 2016.

Band 55: Martina Schattkowsky (Hg.), Frauen und Reformation. Handlungsfelder - Rollenmuster - Engagement, 2016.

Bausteine aus dem Institut für Sächsische Geschichte und Volkskunde. Kleine Schriften zur sächsischen Geschichte und Volkskunde, hrsg. von Enno Bünz, Ira Spieker, Winfried Müller und Martina Schattkowsky, Dresden: Thelem.

Band 34: Julia Kahleyss, Die Kirchenrechnungen der Zwickauer Kirche St. Marien (1441-1534). Edition und Analyse ausgewählter Rechnungen, 2016.

Band 35: Uta Bretschneider/Sönke Friedreich/Ira Spieker (Hg.), Verordnete Nachbarschaften. Transformationsprozesse im deutsch-polnisch-tschechischen Grenzraum seit dem Zweiten Weltkrieg, 2016.

Quellen und Materialien zur sächsischen Geschichte und Volkskunde, hrsg. von Enno Bünz, Ira Spieker, Winfried Müller und Martina Schattkowsky, Leipzig: Leipziger Universitätsverlag:

Band 3, 2: Jens Klingner (Bearb. und Hg.), Die Korrespondenz der Herzogin Elisabeth von Sachsen und ergänzende Quellen, Band 2: Die Jahre 1533 und 1534, 2016.

Spurensuche. Geschichte und Kultur Sachsens. Im Auftrag des Instituts für Sächsische Geschichte und Volkskunde e. V. hrsg. von Enno Bünz, Winfried Müller, Martina Schattkowsky und Ira Spieker, Dresden: Thelem:

Band 6: Enno Bünz/Winfried Müller/Martina SchattKowsky/Ira Spieker (Hg.), Sachsen: weltoffen! Mobilität - Fremdheit - Toleranz, 2016.

\section{Online-Publikationen (Weiterfübrung)}

Sachsen: Weltoffen! Verantwortlich: Ira Spieker, URL: http://www.isgv.de/aktuelles/ sachsen-weltoffen

Digitales Historisches Ortsverzeichnis von Sachsen (DHOV). Verantwortlich: Ulrike Siewert, Lutz Vogel (bis 6/2016), Frank Metasch, URL: http://hov.isgv.de/orte/

Repertorium Saxonicum. Verantwortlich: Ulrike Siewert, Lutz Vogel (bis 6/2016), Frank Metasch, URL: http://www.isgv.de/repsax/

Der Codex diplomaticus Saxoniae im Internet. Verantwortlich: Ulrike Siewert, URL: http://www.isgv.de/codex/

Sächsische Biografie. Verantwortlich: Martina Schattkowsky, URL: http://saebi.isgv. de/ 
Sächsische Gerichtsbücher. Verantwortlich: Volker Jäger (Sächsisches Staatsarchiv Staatsarchiv Leipzig), Martina Schattkowsky, URL: http://www.saechsische-gerichtsbuecher.de/projekt/

Die Elbe. Fluss ohne Grenzen (1815-2015). Verantwortlich: Andreas Martin, URL: http://elbe.isgv.de/

Fremde - Heimat - Sachsen: Vertriebene als Neubauern. Staatliche Integrationsmaßnahmen und individuelle Adaptionsstrategien. Verantwortlich: Ira Spieker, Sönke Friedreich, Uta Bretschneider, URL: www.neubauern-sachsen.de

Erschließung und Digitalisierung des Nachlasses Adolf Spamer. Verantwortlich: Ira Spieker, URL: http://www.isgv.de/projekte/volkskunde/erschliessung-und-digitalisie rung

Sachsen.digital: Interdisziplinäre Wissensplattform zur Geschichte, Kultur und Landeskunde Sachsens, gemeinsames Internetportal der Sächsischen Landesbibliothek Staats- und Universitätsbibliothek Dresden und des ISGV. Verantwortlich: Martina Schattkowsky, Ira Spieker, Ludwig Felber, Michael Schmidt, URL: www.sachsen digital.de

Lebensgeschichtliches Archiv für Sachsen. Verantwortlich: Ira Spieker, URL: http:// lga.isgv.de

Visuelle Quellen zur Volkskultur in Sachsen. Das Bildarchiv des ISGV. Verantwortlich: Andreas Martin, URL: http://bild.isgv.de/ 